\title{
MicroRNA-623 inhibits tumor progression and is a predictor of poor prognosis of breast cancer
}

\author{
CHUNFENG WANG ${ }^{1 *}$, JUAN WANG $^{2 *}$, JING ZHANG $^{1}$, YONGXIANG LI ${ }^{3}$, \\ QINGHUI SUN ${ }^{1}$, FENG GUO $^{1}$ and XIUPENG AN ${ }^{1}$ \\ ${ }^{1}$ Department of Thyroid and Breast Surgery, The Second People's Hospital of Liaocheng, \\ The Second Hospital of Liaocheng Affiliated to Shandong First Medical University; \\ ${ }^{2}$ Department of Hematology and Oncology, The People's Hospital of Linqing; ${ }^{3}$ Department of Emergency, \\ The Second People's Hospital of Liaocheng, The Second Hospital of Liaocheng Affiliated to \\ Shandong First Medical University, Linqing of Liaocheng, Shandong 252600, P.R. China
}

Received April 26, 2020; Accepted October 8, 2020

DOI: $10.3892 / \mathrm{ol} .2020 .12249$

\begin{abstract}
Dysregulated microRNAs (miRNAs) serve vital roles in the progression and prognosis of breast cancer. miR-623 has been reported to influence the progression of numerous other cancers, such as lung adenocarcinoma and hepatocellular carcinoma, however, its role in breast cancer remains unclear. In the present study, the mRNA expression of miR-623 was studied in 121 pairs of breast cancer and adjacent normal tissues and cultured cell lines by reverse-transcription quantitative PCR. The association between miR-623 expression and clinical characteristics or the overall survival rate of patients was investigated by the $\chi^{2}$ test or Cox regression analysis, respectively. The role of miR-623 in cell proliferation, migration and invasion of breast cancer cells was evaluated by cell transfection to regulate miR-623 expression and the CCK8 and Transwell assays, respectively. miR-623 was downregulated in breast cancer tissues and cell lines compared with normal tissues and breast epithelial cell lines. The $\chi^{2}$ test demonstrated that the downregulation of miR-623 was associated with the tumor node metastasis (TNM) stage of patients with breast cancer. miR-623 and TNM stage were considered as two independent prognostic factors for breast cancer. Additionally, cell proliferation, migration, and invasion of breast cancer cells were promoted by the downregulation
\end{abstract}

Correspondence to: Dr Xiupeng An, Department of Thyroid and Breast Surgery, The Second People's Hospital of Liaocheng, The Second Hospital of Liaocheng Affiliated to Shandong First Medical University, 306 Jiankang Road, Linqing of Liaocheng, Shandong 252600, P.R. China

E-mail: anxiupeng921@163.com

*Contributed equally

Key words: breast cancer, microRNA-623, proliferation, migration, invasion, prognosis of miR-623, while upregulation of miR-623 led to inhibition of the aforementioned processes. Downregulation of miR-623 in breast cancer is associated with the development of breast cancer and indicates a poor prognosis of patients. The downregulation of miR-623 promotes cell proliferation, migration and invasion of breast cancer. The findings of the present study indicate that miR-623 functions as a prognosis biomarker and a tumor suppressor in breast cancer, which provides a potential therapeutic target for patients with breast cancer.

\section{Introduction}

Although the management and therapy of cancers has improved in the past decades, cancer remains one of the leading causes of death worldwide, especially breast cancer among women $(1,2)$. Globally, over 1,000,000 people are diagnosed with breast cancer annually and 400,000 females died from breast cancer every year (3). In China, the incidence of breast cancer among women was $20-30 \%$ in 2016 and annually grows by $3-5 \%$ according to Chinese urban cancer registries (4). The number of patients newly diagnosed with breast cancer is still increasing annually, while the therapeutic effect of breast cancer is unsatisfactory $(5,6)$. Distant metastasis and recurrence are the main factors that result in death and poor prognosis of patients with breast cancer (7). Therefore, an improved understanding of tumor progression in breast cancer and the identification of novel biomarkers, which are associated with the development of breast cancer are essential for improved disease stratification and clinical management choices.

Studies have demonstrated that microRNA (miRNA) expression profiling studies have provided a lot of evidence for the regulatory role of miRNAs in tumor progression (8-10). miRNAs are a group of small non-coding RNAs, which are involved in tumorgenesis and regulate a variety of cellular pathways including proliferation, differentiation, migration, and invasion $(11,12)$. miRNA expression profiling can screen dysregulated miRNAs in various cancers, such as prostate cancer, hepatocellular carcinoma, and gastric cancer, which 
provides potential functional miRNAs for the diagnosis and prognosis of cancers (13). Differentially expressed miRNAs being expressed in distinguishable patterns allows them to be used as potentially novel clinical and prognostic biomarkers (14). Downregulation of miR-143 can promote cell apoptosis and regulate the progression of pancreatic cancer (15). miR-558 serves as a biomarker for gastric cancer as its upregulation promotes tumorigenesis and aggressiveness of gastric cancer by targeting heparinase (16). miR-425, miR-132, miR-145 have been reported to serve roles in the progression of breast cancer with different degrees of dysregulation reported (17-19).

miR-623 is a demonstrated downregulated miRNA in breast cancer and has also been reported to serve roles in a number of other cancers, such as gastric cancer, pancreatic cancer, and lung adenocarcinoma (20-22). Reduced or increased expression of miR-623 regulates the progression of various cancers; hence, it was hypothesized that the dysregulation of miR-623 may act as a regulator during the development of breast cancer. The present study aimed to estimate the clinical significance and functional role of miR-623 in breast cancer.

\section{Materials and methods}

Patients and samples. A total of 121 paired samples were used the present study, which included breast cancer tissues and adjacent normal tissues ( $>5 \mathrm{~cm}$ from tumor tissues) with histopathological diagnosis. The inclusion criteria for the patients were: i) Female confirmed diagnosed of breast cancer; ii) underwent mastectomy or breast-conserving surgery; and iii) complete clinical data and follow-up status. The exclusion criteria were as follows: i) Patients underwent chemotherapy, radiotherapy or other types of anticancer therapy; ii) diagnosis with other malignant tumor; and iii) family history of breast cancer. Patients had an average age range of 34-66 years with an average age of $50.18 \pm 6.82$ years. All samples were obtained from patients with breast cancer who underwent surgery at The Second Hospital of Liaocheng affiliated to Shandong First Medical University (Linqing, China) from January 2011 to December 2013. The characteristics of patients are summarized in Table I. The TNM stage was recorded according to the 2010 tumor-node metastasis classification recommended by the American Joint Committee on Cancer (AJCC 7th edition) (23). Samples were immediately frozen in liquid nitrogen and stored at $-80^{\circ} \mathrm{C}$ for subsequent experimentation and analysis. Written informed consent was obtained from every patient, and the present study was approved by the Ethics Committee of The Second Hospital of Liaocheng affiliated to Shandong First Medical University (approval no. 201033). In addition, all patients participated in a 5-year follow-up survey for the collection of the survival information. Patients were followed-up at $6,9,12,15,18,21,24,30,36,42,48$, and 60 months after surgery over the telephone.

Cell culture and transfection. Four human breast cancer cell lines, MCF-7, MDA-MB-231, HCC1954, and HCC1937, and the normal human breast epithelial cell line MCF-10A were used in the present study. All cell lines were purchased from Shanghai Cell Bank of the Chinese Academy of Medical Sciences and cultured in Dulbecco's modified Eagle's medium
(DMEM) supplemented with $10 \%$ fetal bovine serum (FBS) (both Gibco; Thermo Fisher Scientific Inc.). All cell cultures were maintained at $37^{\circ} \mathrm{C}$ in a humidified incubator with $5 \% \mathrm{CO}_{2}$ for $24 \mathrm{~h}$.

To regulate the expression of miR-623 and explore its effects on the cellular processes of breast cancer, breast cancer cells were transfected with $20 \mathrm{nM}$ miR-623 mimic, miR-623 inhibitor, mimic negative control (mimic NC), or inhibitor negative control (inhibitor NC) purchased from Guangzhou RiboBio Co., Ltd. Lipofectamine $2000^{\circledR}$ reagent (Invitrogen; Thermo Fisher Scientific Inc.) was used for transfection at $37^{\circ} \mathrm{C}$ for $24 \mathrm{~h}$. Untransfected cells were defined as control group. The sequences of transfections are as follows: miR-623 mimic, 5'-AUCCCUUGCAGGGGCUGUUGGGU-3'; miR-623 inhibitor, 5'-ACCCAACAGCCCCUGCAAGGGAU-3'; mimic NC, 5'-UUCUCCGAACGUGUCACGUTTACGUGACAC GUUCGGAGAATT-3' and inhibitor NC, 5'-CAGUACUUU UGUGUAGUACAA-3'. After 48 h of transfection, subsequent experimentation was performed.

$R N A$ isolation and reverse-transcription quantitative (RT-q) $P C R$ assay. Total RNA from collected tissues and cultured cells was extracted by using TRIzol reagent (Invitrogen; Thermo Fisher Scientific Inc.) and reverse transcribed into cDNA by using miRNA First-Strand cDNA Synthesis kit (Invitrogen; Thermo Fisher Scientific Inc.). The reverse transcription protocol was: $37^{\circ} \mathrm{C}$ for $1 \mathrm{~h}$ and $85^{\circ} \mathrm{C}$ for $5 \mathrm{~min}$. SYBR Green I Master Mix kit (Invitrogen; Thermo Fisher Scientific Inc.) was used to perform RT-qPCR on the 7300 Real-Time PCR System (Applied Biosystems; Thermo Fisher Scientific Inc.). The thermocycling conditions were as follows: $95^{\circ} \mathrm{C}$ for $10 \mathrm{~min}$ followed by 40 cycles of $95^{\circ} \mathrm{C}$ for $15 \mathrm{sec}$ and $60^{\circ} \mathrm{C}$ for $15 \mathrm{sec}$. The expression of miR-623 was normalized to that of U6 with relative quantification by the $2^{-\Delta \Delta \mathrm{Cq}}$ calculation method (24). The primer sequences of miR-623 were: Forward. 5'-ATCCCT TGCAGGGGCTGTTGGGT-3' and reverse, 5'-GCCAGCACA GAATTAATACGAC-3'. The primer sequences of U6 were: Forward 5'-CTCGCTTCGGCAGCACA-3'; reverse 5'-AAC GCTTCACGAATTTGCGT-3'.

CCK8 assay. To measure the proliferation ability of breast cancer cells, a CCK8 assay was performed according to the manufacturer's instructions. Cells were plated in 96-well plates at a density of $5 \times 10^{3}$ cells/well and cultured at $37^{\circ} \mathrm{C}$ with $5 \% \mathrm{CO}_{2}$ for $0,24,48$ and $72 \mathrm{~h}$. Cells were then incubated with $10 \mu \mathrm{l}$ cell counting kit-8 (CCK-8) reagent (Dojindo Molecular Technologies Inc.) per well for $4 \mathrm{~h}$ at $37^{\circ} \mathrm{C}$ with $5 \% \mathrm{CO}_{2}$. Absorbance at $450 \mathrm{~nm}$ was measured with a microplate reader (Thermo Fisher Scientific Inc.).

Transwell assay. Matrigel-uncoated and coated transwell (for invasion) inserts (8-mm pore size; Thermo Fisher Scientific Inc.) were used for the detection of cell migration and invasion. Matrigel precoating was performed at $37^{\circ} \mathrm{C}$ for $1 \mathrm{~h}$ for the invasion assay. A total of $2 \times 10^{5}$ transfected cells were seeded into the upper chamber with serum-free medium and culture medium with $10 \%$ FBS was placed in the lower chamber as a chemoattractant. The Transwell was incubated at $37^{\circ} \mathrm{C}$ for $24 \mathrm{~h}$, after that, transwells were removed and stained with $0.1 \%$ crystal violet (Sigma; Merck KGaA) at $37^{\circ} \mathrm{C}$ 
Table I. Association between miR-623 expression and the characteristics of patients with breast cancer.

\begin{tabular}{|c|c|c|c|c|}
\hline \multirow[b]{2}{*}{ Characteristics } & \multirow[b]{2}{*}{ Patients $(n=121)$} & \multicolumn{2}{|c|}{ miR-623 expression } & \multirow[b]{2}{*}{ P-value } \\
\hline & & Low $(n=68)$ & $\operatorname{High}(n=53)$ & \\
\hline Age, years & & & & 0.162 \\
\hline$\leq 50$ & 54 & 30 & 24 & \\
\hline$>50$ & 67 & 38 & 29 & \\
\hline Tumor size, $\mathrm{cm}$ & & & & 0.247 \\
\hline$\leq 5$ & 62 & 32 & 30 & \\
\hline$>5$ & 59 & 36 & 23 & \\
\hline Lymph node metastasis & & & & 0.162 \\
\hline Negative & 69 & 40 & 29 & \\
\hline Positive & 52 & 28 & 24 & \\
\hline TNM stage & & & & 0.028 \\
\hline I-II & 71 & 36 & 35 & \\
\hline III-IV & 50 & 32 & 18 & \\
\hline HER 2 status & & & & 0.255 \\
\hline Negative & 55 & 29 & 26 & \\
\hline Positive & 66 & 39 & 27 & \\
\hline ER status & & & & 0.341 \\
\hline Negative & 58 & 35 & 23 & \\
\hline Positive & 63 & 33 & 30 & \\
\hline PR status & & & & 0.276 \\
\hline Negative & 57 & 37 & 23 & \\
\hline Positive & 64 & 31 & 33 & \\
\hline
\end{tabular}

HER 2, human epidermal growth factor receptor 2; ER, estrogen receptor; PR, progesterone receptor; TNM, tumor node metastases.

A

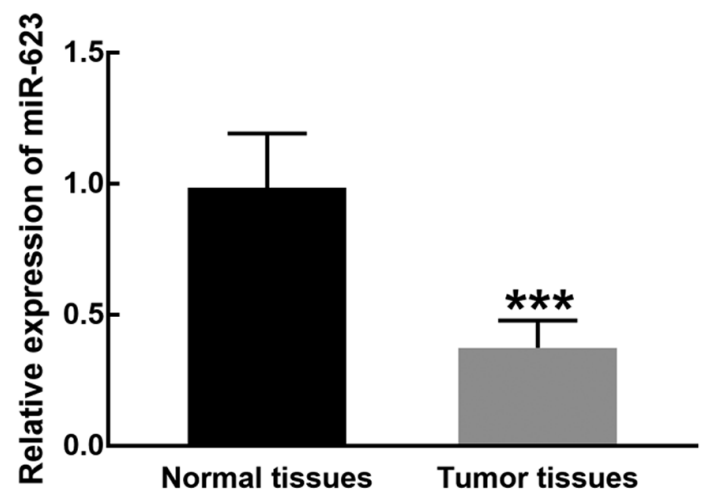

B

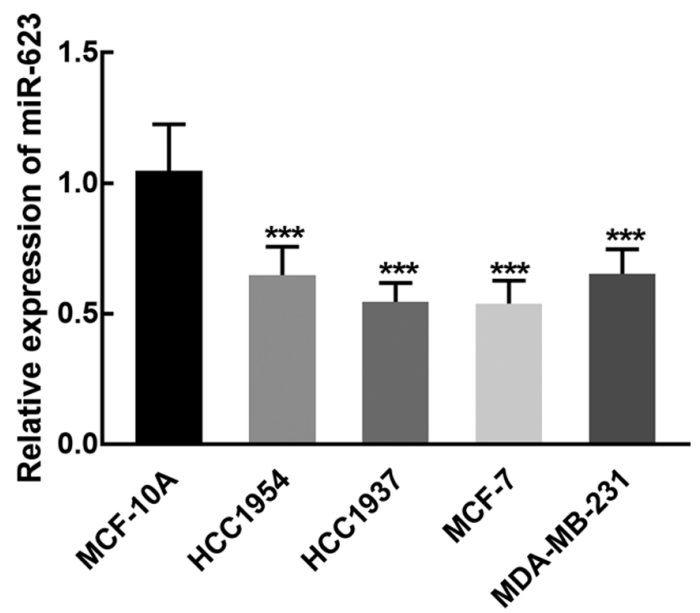

Figure 1. miR-623 is downregulated in breast cancer tissues and cell lines. (A) Relative expression of miR-623 in 121 paired breast cancer and adjacent normal tissues detected by reverse-transcription quantitative PCR. ${ }^{* * *} \mathrm{P}<0.001$ compared with normal tissues. (B) Relative expression of miR-623 in breast cancer cell lines (HCC1954, HCC1937 MCF-7, MDA-MB-231) and a normal human breast epithelial cell line MCF-10A. ${ }^{* * *} \mathrm{P}<0.001$ compared with MCF-10A. miR, microRNA.

for 5 min. The number of migrated and invaded cells in the lower chamber was detected by a fluorescence microscope (magnification, x100).
Statistical analysis. All data were represented as the mean value \pm SD obtained from 3 repeats and were analyzed by SPSS 20.0 software (IBM Corp.) and GraphPad Prism 5.0 software 
(GraphPad Software, Inc.) The differences in the expression of miR-623 in breast cancer tissues and adjacent normal tissues were analyzed using a paired Student's t-test. The differences between multiple groups was assessed by one-way ANOVA followed by the post hoc Tukey's test. The association between miR-623 expression and clinical characteristics of patients was evaluated by the $\chi^{2}$ test. The survival curves of patients were generated using the Kaplan-Meier method and were compared using the log-rank test. Additionally, the prognostic value of miR-623 was further assessed by the Cox regression analyses. $\mathrm{P}<0.05$ was considered to indicate a statistically significant difference.

\section{Results}

Expression level of miR-623 in breast cancer tissue and cells. In the collected breast cancer and adjacent normal tissues, the expression of miR-623 was detected by RT-qPCR. miR-623 demonstrated significantly decreased expression in breast cancer tissues compared with adjacent normal tissues $(\mathrm{P}<0.001$; Fig. 1A). Similarly, miR-623 was significantly downregulated in breast cancer cell lines (HCC1954, HCC1937, MCF-7 and MDA-MB-231) compared with MCF-10A cells $(\mathrm{P}<0.001$; Fig. 1B). The relatively lower expression of miR-623 in HCC1937 and MCF-7 cells compared to the other cancer cell lines indicated that they are more sensitive to the dysregulation of miR-623, hence they were chosen for subsequent cell experiments.

Association between miR-623 expression and clinical characteristics of patients with breast cancer. A total of 121 breast cancer patients were divided into miR-623 high or low expression groups based on the mean value of the miR-623 level (0.375) in breast cancer tissues. Results of the $\chi^{2}$ test demonstrated a significant association between miR-623 expression and the TNM stage of patients $(\mathrm{P}=0.028)$, while age, tumor size, lymph node metastasis, and the status of human epidermal growth factor receptor 2, estrogen receptor (ER) and progesterone receptor of patients demonstrated no significant association with the expression of miR-623 $(\mathrm{P}>0.05$, Table I).

Prognostic value of miR-623 in breast cancer. Fig. 2 demonstrates the survival rate of patients with breast cancer with low or high miR-623 expression levels 5 years after surgery. Patients with low miR-623 expression demonstrated a shorter overall survival time compared with patients with high miR-623 expression (Log-rank P=0.020; Fig. 2). Additionally, the results of Cox regression analysis indicated that miR-623 expression [hazard ratio (HR) factor $=2.743$; 95\% confidence interval $(\mathrm{CI})=1.260-5.971 ; \mathrm{P}=0.011]$ and $\mathrm{TNM}$ stage (HR factor $=2.191 ; 95 \% \mathrm{CI}=1.082-4.434 ; \mathrm{P}=0.029)$ are two independent prognostic factors for breast cancer due to their close association with the survival rate of patients (Table II).

Effect of miR-623 expression on cell proliferation of breast cancer cells. miR-623 mimic, miR-623 inhibitor and corresponding negative controls were transfected into HCC1937 and MCF-7 cells for the overexpression or knockdown of miR-623. In addition, the proliferation ability of transfected
Table II. Cox regression analysis of the association between characteristics of patients with breast cancer and survival rate.

\begin{tabular}{lccc}
\hline Characteristics & HR factor & $95 \%$ CI & P-value \\
\hline miR-623 expression & 2.743 & $1.260-5.971$ & 0.011 \\
Age & 1.250 & $0.602-2.598$ & 0.549 \\
Tumor size & 1.633 & $0.789-3.377$ & 0.186 \\
Lymph node metastasis & 1.694 & $0.831-3.453$ & 0.147 \\
TNM stage & 2.191 & $1.082-4.434$ & 0.029 \\
HER2 status & 1.594 & $0.765-3.320$ & 0.213 \\
ER status & 1.447 & $0.698-3.003$ & 0.321 \\
PR status & 1.504 & $0.741-3.049$ & 0.258 \\
\hline
\end{tabular}

HER 2, human epidermal growth factor receptor 2; ER, estrogen receptor; $\mathrm{PR}$, progesterone receptor; $\mathrm{HR}$, hazard ratio; $\mathrm{CI}$, confidence interval.

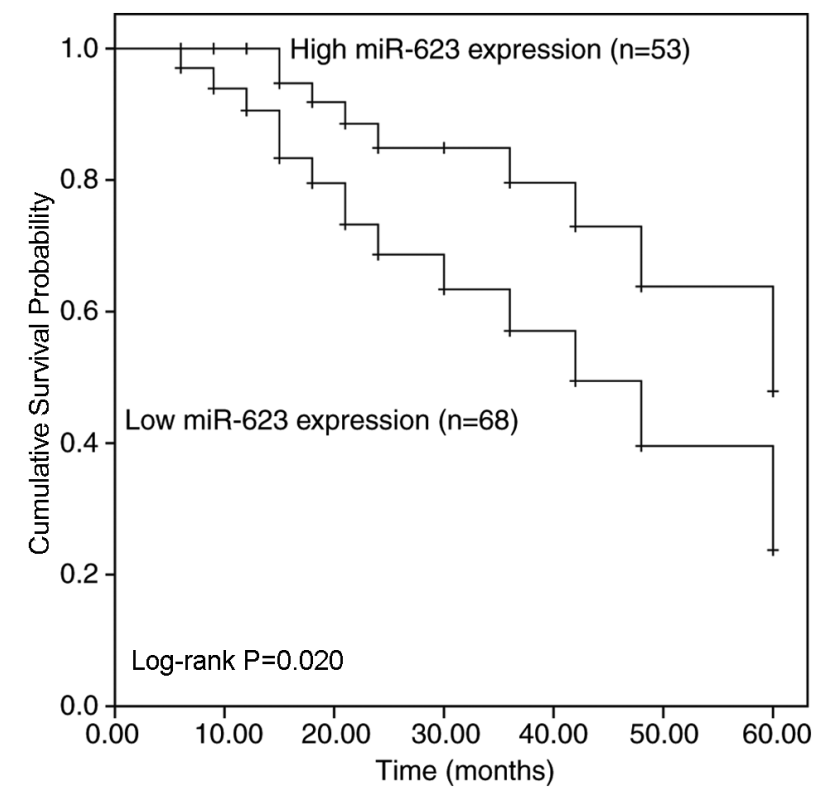

Figure 2. Kaplan-Meier curve of patients with breast cancer with different miR-623 expression levels. Low miR-623 expression predicts a poor prognosis of patients with breast cancer (Log-rank $\mathrm{P}=0.020$ relative to patients with high miR-623 expression). miR, microRNA.

cells was analyzed by the CCK8 assay to investigate the effect of miR-623 expression on cell proliferation of breast cancer cells. Following the transfection of miR-623 mimic, miR-623 was significantly upregulated, while the transfection of miR-623 inhibitor significantly downregulated miR-623 in HCC1937 and MCF-7 cells compared with cells in control group $(\mathrm{P}<0.001$; Fig. $3 \mathrm{~A})$.

The results of CCK8 assay revealed that the proliferation of HCC1937 and MCF-7 cells was significantly inhibited by the miR-623 mimic and promoted by the miR-623 inhibitor $(\mathrm{P}<0.05$ relative to control group; Fig. 3B). This suggests that miR-623 may be involved in the proliferation of breast cancer cells.

Role of miR-623 in cell migration and invasion of breast cancer cells. Transwell assays were performed to evaluate the 

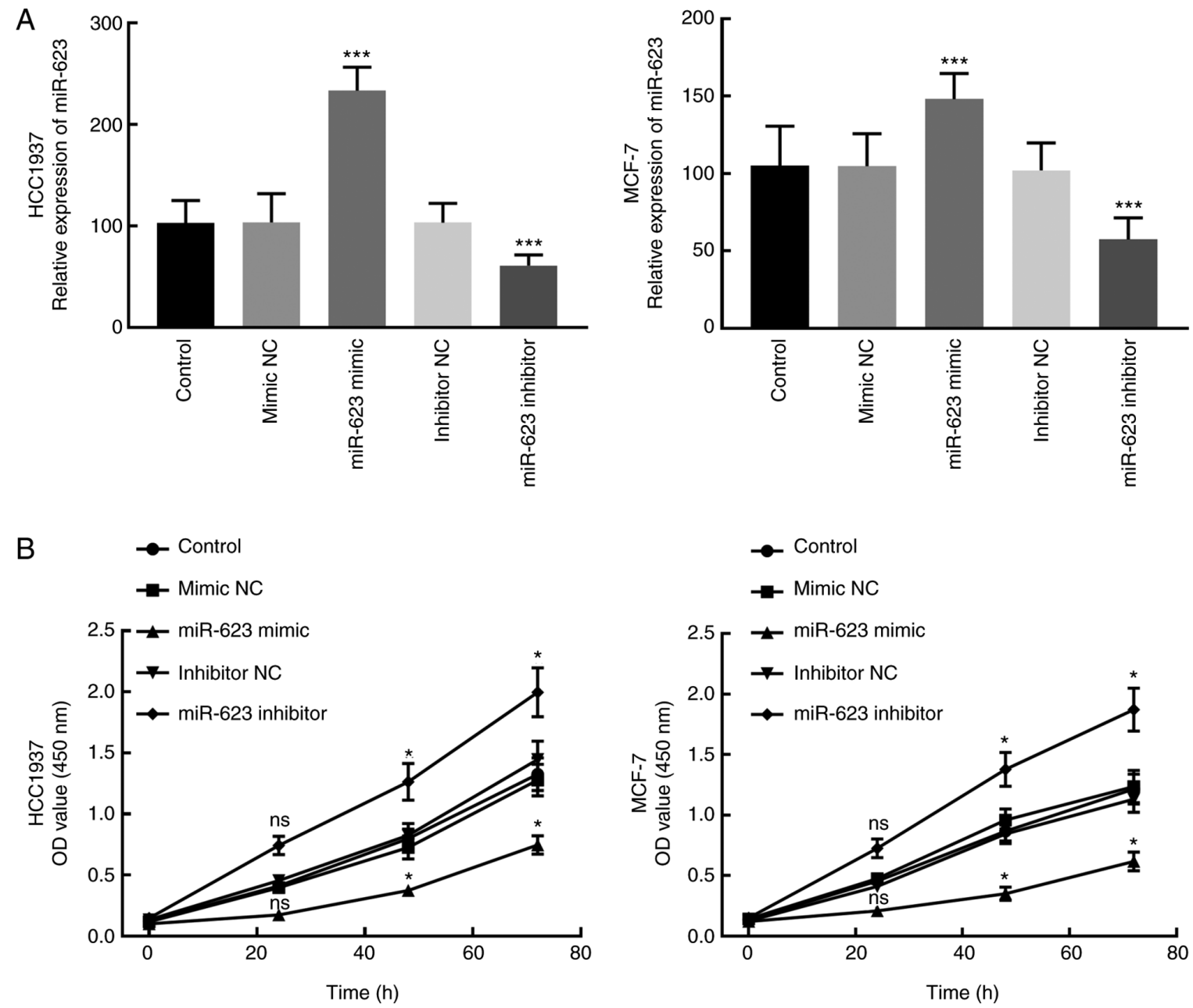

Figure 3. Downregulation of miR-623 promotes cell proliferation of HCC1937 and MCF-7. (A) Relative expression of miR-623 in HCC1937 and MCF-7 cells transfected with miR-623 mimic, miR-623 inhibitor and corresponding NC. ${ }^{* * *} \mathrm{P}<0.001$ compared with Control. (B) Proliferation of HCC11937 and MCF-7 cells after transfection. "P<0.05 compared with Control. miR, microRNA; control, untransfected cells; NC, negative control; OD, optical density; ns, not significant.

migration and invasion of HCC1937 and MCF-7 cells with different expression levels of miR-623. The results demonstrated that the migration and invasion of cells transfected with the miR-623 mimic was significantly lower compared with cells transfected with miR-623 inhibitor, which revealed the inhibitory role of miR-623 in the migration and invasion of breast cancer cells $(\mathrm{P}<0.001$; Fig. $4 \mathrm{~A}$ and $\mathrm{B})$.

\section{Discussion}

Due to the increasing number of new cases and the death rate resulting from recurrence and metastasis, breast cancer is still considered as the most malignant tumor in women (25). The exploration of novel biomarkers in breast cancer has received increasing attention in recent decades (26). miRNAs have been identified as biomarkers for the diagnosis or prognosis of various cancers, such as gastric cancer, lung cancer, colorectal cancer, and numerous other cancers and diseases, such as acute myeloid leukemia and Alzheimer's disease (16,27-29). For example, overexpressed miR-675 in non-small cell lung cancer promotes the progression of the disease by activating the NF- $\kappa \mathrm{B}$ signaling pathway (30). A number of miRNAs, such as miR-145-5p, miR-940, and miR-205-3p (19,31,32), which serve roles in the progression of breast cancer have been considered as potential biomarkers for breast cancer.

Previously, miR-623 was reported to be dysregulated in breast cancer (33) and to serve roles in various cancers (20-22). For example, miR-623 was demonstrated to inhibit cell migration, invasion, and metastasis of pancreatic cancer in vitro and in vivo by directly targeting MMP1 (22). In gastric cancer, miR-623 can function as a tumor suppressor and enhance chemosensitivity (20). miR-623 has also been demonstrated to suppress the progression of lung adenocarcinoma by targeting MMP-2 and MMP-9 (21). The present study was performed to identify the role of miR-623 in the progression and prognosis of breast cancer. miR-623 was found to be downregulated in breast cancer tissues and cell lines compared with normal breast tissues and cell lines. In the present study, overexpression of miR- 623 by miR- 623 mimic transfection was demonstrated to exert inhibitory effects on cell proliferation, migration and invasion of breast cancer, indicating the tumor suppressor role of miR-623 in the progression of breast cancer.

Recently, the biological function of miR-623 has been revealed by Li et al (34) in MCF-7 and MDA-MB-453 cells. The exogenous overexpression of miR-623 inhibited cell 

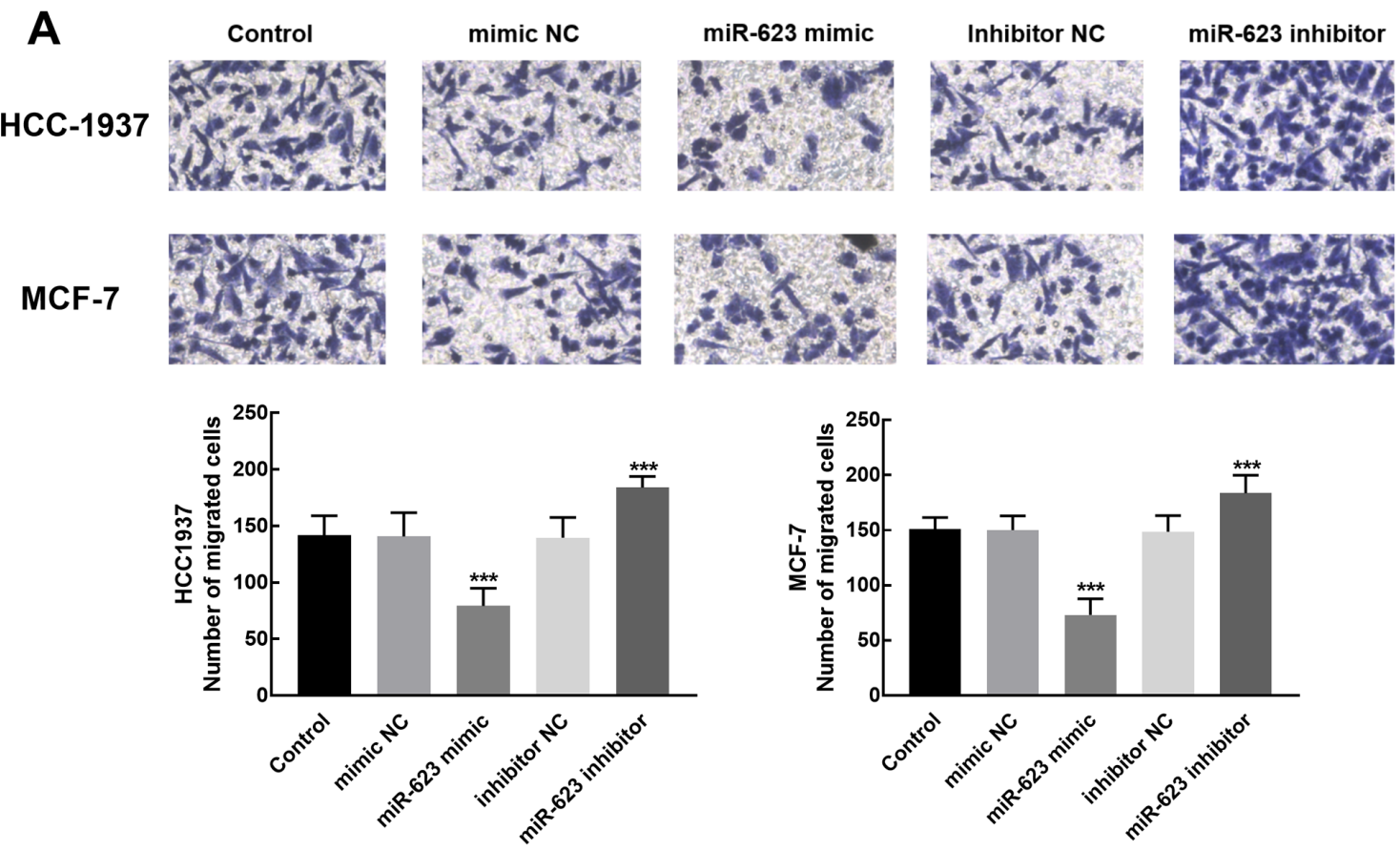

B
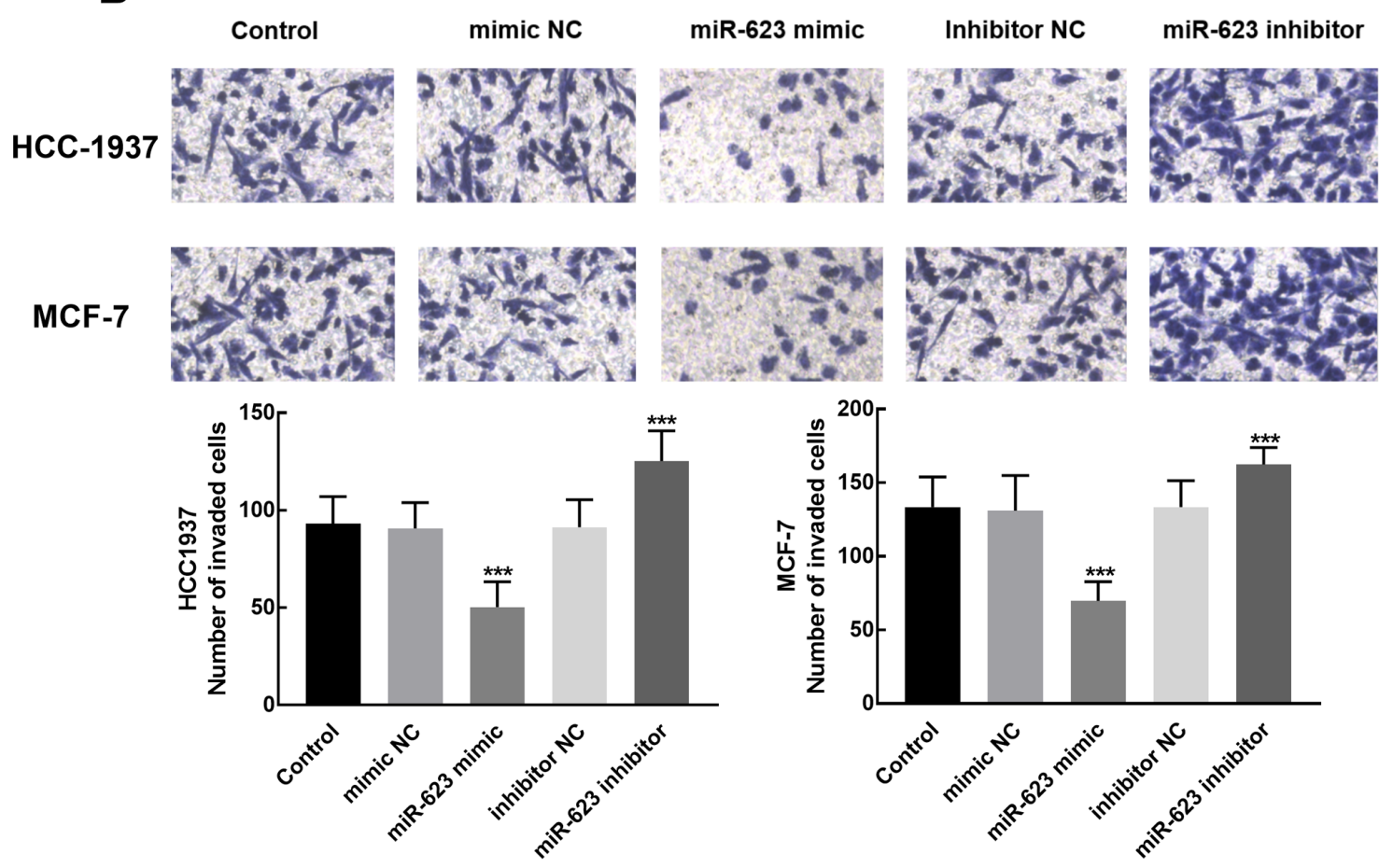

Figure 4. Downregulation of miR-623 promotes cell migration and invasion of HCC1937 and MCF-7. (A) Migration of HCC1937 and MCF-7 after the transfection of miR-623 mimic, miR-623 inhibitor and corresponding NC. ${ }^{* * *} \mathrm{P}<0.001$ compared with control. (B) Invasion of HCC1937 and MCF-7 after the transfection of miR-623 mimic, miR-623 inhibitor and corresponding NC. ${ }^{* * * *} \mathrm{P}<0.001$ compared control. miR, microRNA; control, untransfected cells; NC, negative control.

proliferation and promoted cell apoptosis of breast cancer by targeting X-ray repair cross-complementing protein 5 (34), which is consistent with the biological function of miR-623 in MCF-7 and HCC1937 cells in the present study. In the present study, in addition, to miR-623 function in cell progression of breast cancer, the clinical significance of miR-623 was also assessed. miR-623 expression had a close relationship with the TNM stage of patients. Additionally, in the present study patients who had low miR-623 mRNA expression demonstrated poorer prognosis compared with patients with high miR-623 expression, indicating that the downregulation of miR-623 was associated with poor prognosis of breast cancer. Based on the findings of the present study, miR-623 expression and TNM stage were considered as two independent prognostic factors for breast cancer. miRNAs have been reported to predict the prognosis of a variety of cancers and serve as independent 
prognostic factors for cancers. For instance, overexpression of miR-153 in prostate cancer can predicate the poor prognosis of patients (35). Downregulation of miR-1247-5p in breast cancer is associated with poor prognosis of patients (36). All the aforementioned findings of the present study, indicate that miR-623 participated in and suppressed the progression of breast cancer.

However, in addition to the investigation of the function of miR-623 in the progression of breast cancer, it is also necessary to determine the mechanism underlying the functional role of miR-623. Previously, MMP1 and Cyclin D1 were demonstrated to be direct targets of miR-623, regulating how miR-623 participates in the progression of pancreatic cancer and gastric cancer $(20,22)$. Hence, it was speculated that miR-623 may regulate the progression of breast cancer by targeting these genes or other potential targets, which is a limitation of this study and needs further experiments to validate this hypothesis.

In conclusion, the results of the present study demonstrated that miR-623 was downregulated in breast cancer tissues and cells, which was associated with the TNM stage of patients and predicted poor prognosis of patients with breast cancer. miR-623 participated in the progression of breast cancer and was an independent prognostic factor together with TNM stage. In addition, knocking down of miR-623 significantly promoted cell proliferation, migration and invasion of breast cancer, while its overexpression significantly suppressed these cellular processes, which indicated the tumor inhibitor role of miR-623 in breast cancer. Malignant tumor is a multifaceted disease with important differences among diverse types of cancers (37). This research is an expanding study that applied regular methods to investigate the role of miR-623 in breast cancer.

\section{Acknowledgements}

Not applicable.

\section{Funding}

No funding was received.

\section{Availability of data and materials}

The data used during the current study are available from the corresponding author on reasonable request.

\section{Authors' contributions}

CW, JW, JZ, YL, QS, FG and XA made substantial contributions to conception and design. CW, JW, JZ, YL, QS and FG contributed to acquisition of data. $\mathrm{CW}$ and $\mathrm{XA}$ analyzed and interpreted the data. All the authors were involved in writing the manuscript. All authors have read and approved the manuscript.

\section{Ethics approval and consent to participate}

Written informed consent was obtained from every patient and this study was approved by the Ethics Committees of The Second Hospital of Liaocheng affiliated to Shandong First Medical University (Linqing, China) (approval number, 201033).

\section{Patient consent for publication}

Not applicable.

\section{Competing interests}

The authors declare that they have no competing interests.

\section{References}

1. Espie M: The management of breast cancer. Diagn Interv Imaging 95: 753-757, 2014.

2. Shimizu C: Breast cancer in young women: Its biological and clinical uniqueness and needs of comprehensive care. Breast Cancer 21: 641-642, 2014.

3. Jemal A, Bray F, Center MM, Ferlay J, Ward E and Forman D: Global cancer statistics. CA Cancer J Clin 61: 69-90, 2011.

4. Li T, Mello-Thoms C and Brennan PC: Descriptive epidemiology of breast cancer in China: Incidence, mortality, survival and prevalence. Breast Cancer Res Treat 159: 395-406, 2016.

5. Sorscher S: Prognosis of patients with multifocal breast cancer. Hum Pathol 84: 335-336, 2019.

6. Plichta JK: Breast cancer prognostic staging and internal mammary lymph node metastases: A brief overview. Chin Clin Oncol 8: S11, 2019.

7. Van Langenberg A: Prognosis after breast recurrence following conservative surgery and radiotherapy in patients with node-negative breast cancer. Br J Surg 87: 681-683, 2000.

8. Hwang J, Min BH, Jang J, Kang SY, Bae H, Jang SS, Kim JI and Kim KM: MicroRNA expression profiles in gastric carcinogenesis. Sci Rep 8: 14393, 2018.

9. Lee YS and Dutta A: MicroRNAs in cancer. Annu Rev Pathol 4: 199-227, 2009.

10. Lu J, Getz G, Miska EA, Alvarez-Saavedra E, Lamb J, Peck D, Sweet-Cordero A, Ebert BL, Mak RH, Ferrando AA, et al: MicroRNA expression profiles classify human cancers. Nature 435: 834-838, 2005.

11. Mohr AM and Mott JL: Overview of microRNA biology. Semin Liver Dis 35: 3-11, 2015.

12. Yates LA, Norbury CJ and Gilbert RJ: The long and short of microRNA. Cell 153: 516-519, 2013.

13. Iorio MV and Croce CM: MicroRNA dysregulation in cancer: Diagnostics, monitoring and therapeutics. A comprehensive review. EMBO Mol Med 4: 143-159, 2012.

14. Kallioniemi A: Molecular signatures of breast cancer-predicting the future. N Engl J Med 347: 2067-2068, 2002.

15. Xu B, Liu J, Xiang X, Liu S, Zhong P, Xie F, Mou T and Lai L: Expression of miRNA-143 in pancreatic cancer and its clinical significance. Cancer Biother Radiopharm 33: 373-379, 2018.

16. Zheng L, Jiao W, Song H, Qu H, Li D, Mei H, Chen Y, Yang F, Li H, Huang K and Tong Q: miRNA-558 promotes gastric cancer progression through attenuating Smad4-mediated repression of heparanase expression. Cell Death Dis 7: e2382, 2016.

17. Xiao S, Zhu H, Luo J, Wu Z and Xie M: miR425-5p is associated with poor prognosis in patients with breast cancer and promotes cancer cell progression by targeting PTEN. Oncol Rep 42: 2550-2560, 2019.

18. Li S, Xu JJ and Zhang QY: MicroRNA-132-3p inhibits tumor malignant progression by regulating lysosomal-associated protein transmembrane 4 beta in breast cancer. Cancer Sci 110: 3098-3109, 2019.

19. Tang W, Zhang X, Tan W, Gao J, Pan L, Ye X, Chen L and Zheng W: miR-145-5p suppresses breast cancer progression by inhibiting SOX2. J Surg Res 236: 278-287, 2019.

20. Jiang L, Yang W, Bian W, Yang H, Wu X, Li Y, Feng W and Liu X: MicroRNA-623 targets cyclin D1 to inhibit cell proliferation and enhance the chemosensitivity of cells to 5-fluorouracil in gastric cancer. Oncol Res 27: 19-27, 2018.

21. Wei S, Zhang ZY, Fu SL, Xie JG, Liu XS, Xu YJ, Zhao JP and Xiong WN: Hsa-miR-623 suppresses tumor progression in human lung adenocarcinoma. Cell Death Dis 7: e2388, 2016.

22. Chen Y, Peng S, Cen H, Lin Y, Huang C, Chen Y, Shan H, Su Y and Zeng L: MicroRNA hsa-miR-623 directly suppresses MMP1 and attenuates IL-8-induced metastasis in pancreatic cancer. Int J Oncol 55: 142-156, 2019. 
23. Edge SB and Compton CC: The American Joint Committee on Cancer: The 7th edition of the AJCC cancer staging manual and the future of TNM. Ann Surg Oncol 17: 1471-1474, 2010.

24. Livak KJ and Schmittgen TD: Analysis of relative gene expression data using real-time quantitative PCR and the 2(-Delta Delta C(T)) method. Methods 25: 402-408, 2001.

25. Bray F, Ferlay J, Soerjomataram I, Siegel RL, Torre LA and Jemal A: Global cancer statistics 2018: GLOBOCAN estimates of incidence and mortality worldwide for 36 cancers in 185 countries. CA Cancer J Clin 68: 394-424, 2018.

26. $\mathrm{Li} \mathrm{G}, \mathrm{Hu} \mathrm{J}$ and $\mathrm{Hu} \mathrm{G}$ : Biomarker studies in early detection and prognosis of breast cancer. Adv Exp Med Biol 1026: 27-39, 2017.

27. Li Q, Wang $\mathrm{Y}, \mathrm{Hu} \mathrm{R}$ and Yang G: Dysregulation of SPRR3/miR-876-3p axis contributes to tumorigenesis in non-small-cell lung cancer. Onco Targets Ther 13: 2411-2419, 2020.

28. Sahami-Fard MH, Kheirandish S and Sheikhha MH: Expression levels of miR-143-3p and -424-5p in colorectal cancer and their clinical significance. Cancer Biomark 24: 291-297, 2019.

29. Zhang Z, Zhang J, Li J, Geng H, Zhou B, Zhang B and Chen H: miR-320/ELF3 axis inhibits the progression of breast cancer via the PI3K/AKT pathway. Oncol Lett 19: 3239-3248, 2020.

30. Feng Y, Yang C, Hu D, Wang X and Liu X: miR-675 promotes disease progression of non-small cell lung cancer via activating NF- $\kappa \mathrm{B}$ signaling pathway. Cell Mol Biol (Noisy-le-grand) 63: 7-10, 2017.

31. Liu W, Xu Y, Guan H and Meng H: Clinical potential of miR-940 as a diagnostic and prognostic biomarker in breast cancer patients. Cancer Biomark 22: 487-493, 2018.
32. Qiu C, Huang F, Zhang Q, Chen W and Zhang H: miR-205-3p promotes proliferation and reduces apoptosis of breast cancer MCF-7 cells and is associated with poor prognosis of breast cancer patients. J Clin Lab Anal 33: e22966, 2019.

33. Hamam R, Ali AM, Alsaleh KA, Kassem M, Alfayez M, Aldahmash A and Alajez NM: microRNA expression profiling on individual breast cancer patients identifies novel panel of circulating microRNA for early detection. Sci Rep 6: 25997, 2016

34. Li Q, Liu J, Jia Y, Li T and Zhang M: miR-623 suppresses cell proliferation, migration and invasion through direct inhibition of XRCC5 in breast cancer. Aging (Albany NY) 12: 10246-10258, 2020.

35. Bi CW, Zhang GY, Bai Y, Zhao B and Yang H: Increased expression of miR-153 predicts poor prognosis for patients with prostate cancer. Medicine (Baltimore) 98: e16705, 2019.

36. Zeng B,Li Y,Feng Y,Lu M, Yuan H, Yi Z,Wu Y, Xiang T, Li H and Ren G: Downregulated miR-1247-5p associates with poor prognosis and facilitates tumor cell growth via DVL1/Wnt/ $\beta$-catenin signaling in breast cancer. Biochem Biophys Res Commun 505: 302-308, 2018.

37. Das J, Gayvert KM and Yu H: Predicting cancer prognosis using functional genomics data sets. Cancer Inform 13 (Suppl 5): S85-S88, 2014.

(i) $\Theta$ This work is licensed under a Creative Commons Attribution-NonCommercial-NoDerivatives 4.0 International (CC BY-NC-ND 4.0) License. 